\title{
Inhaltsübersicht
}

Vorwort $\ldots \ldots \ldots \ldots \ldots \ldots \ldots \ldots \ldots \ldots \ldots \ldots \ldots \ldots \ldots, \quad \mathrm{V}$

\section{Teil 1 Einführung}

I. Rechtsquelle: Das Gerichts- und Notarkostengesetz (GNotKG) $\ldots \ldots \quad 7$

II. Das Kostenschuldverhältnis . . . . . . . . . . . . . . . . 30

III. Vollzugs- und Betreuungstätigkeiten $\ldots \ldots \ldots \ldots \ldots \ldots \ldots \ldots . \ldots 1$

IV. Wichtige Einzelregelungen $\ldots \ldots \ldots \ldots \ldots \ldots \ldots \ldots \ldots \ldots . \ldots \ldots$

V. Die gerichtliche Überprüfung des Kostenanspruchs . . . . . . . . . 93

VI. Übergangsrecht ................................. 119

\section{Teil 2 Grundstückskauf}

I. Überblick ...................................... 136

II. Standardkaufvertrag in den Varianten Direktzahlung und

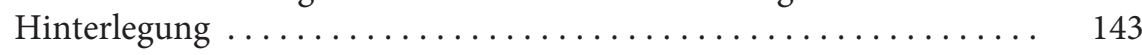

III. Kaufvertrag mit Hinzurechnungsposten und Bebauung auf Rechnung des Erwerbers $\ldots \ldots \ldots \ldots \ldots \ldots \ldots \ldots \ldots \ldots \ldots \ldots$

IV. Kaufvertrag und weitere Erklärungen mit demselben bzw. einem verschiedenen Gegenstand ........................ 176

V. Ausgewählte Kaufvertragstypen $\ldots \ldots \ldots \ldots \ldots \ldots \ldots \ldots \ldots . \ldots \ldots$

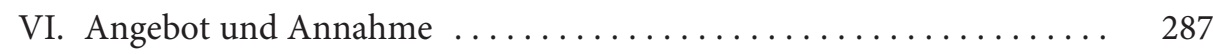

VII. Bedingte Kaufverträge und Optionsrechte . . . . . . . . . . . 313

VIII. Kaufverträge mit Kaufpreis unter dem Verkehrswert .......... 320

IX. Änderungen oder Ergänzungen (Nachträge) .............. 321

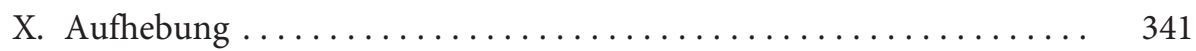

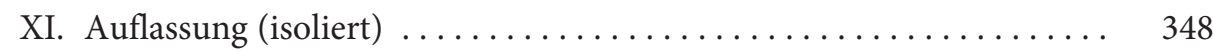

XII. Besondere Regelungsgegenstände ...................... 359

XIII. Tauschvertrag ................................. 379

XIV. Spezielle Vollzugstätigkeit: Erstellen einer XML-Datei .......... 387

\section{Teil 3 Überlassungsverträge}

I. Überblick . . . . . . . . . . . . . . . . . . . . . . . . . . . . . . . . . . . . 395

II. Überlassungsverträge ohne Gegenleistungen (Schenkungen) $\ldots . .4419$

III. Überlassungsverträge mit Gegenleistungen .............. 426 
IV. Zuwendungen in besonderen Gemeinschaftsverhältnissen . . . . . . 443

V. Vertragsänderungen und -aufhebungen .................. 445

VI. Rückabwicklungen und Verfügungsgeschäfte ............. 448

\section{Teil 4 Wohnungs- und Teileigentum}

I. Überblick ......................................... 454

II. Begründung nach $₫ 8$ WEG. . . . . . . . . . . . . . . . . . . 462

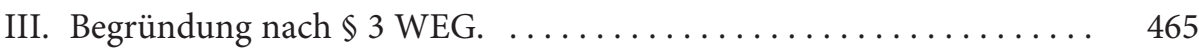

IV. Weitere verschiedene Beurkundungsgegenstände im Rahmen

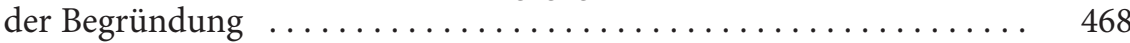

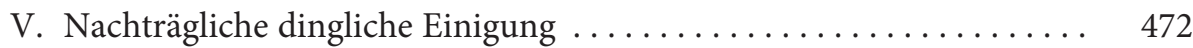

VI. Unterteilung von Wohnungs-/Teileigentum .............. 473

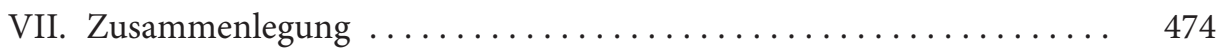

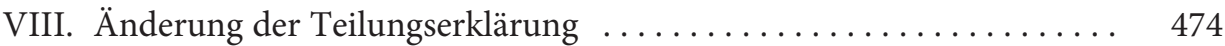

IX. Aufhebung von Wohnungs- bzw. Teileigentum ............. 478

X. Dauerwohn- und Dauernutzungsrecht .................. 482

XI. Verkauf ........................................ 483

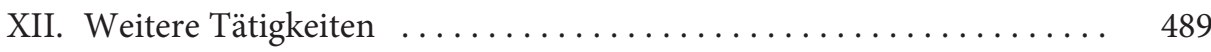

\section{Teil 5 Erbbaurecht}

I. Überblick . . . . . . . . . . . . . . . . . . . . . . . . . . . . . 498

II. Erbbaurechtsbestellung ............................ 504

III. Nachträgliche dingliche Einigung . . . . . . . . . . . . . . 513

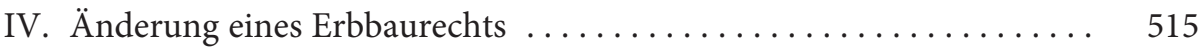

V. Aufhebung eines Erbbaurechts ...................... 518

VI. Verkauf eines Erbbaurechts ... . . . . . . . . . . . . . . . . . . . . . . . 524

VII. Übertragung eines Erbbaurechts ..................... 527

VIII. Weitere Geschäfte . . . . . . . . . . . . . . . . . . . . . . . . . 529

\section{Teil 6 Grundpfandrechte und weitere Sicherungs- geschäfte}

I. Überblick .................................. 541

II. Bestellung durch Beurkundung oder Entwurf mit Unterschriftsbeglaubigung ................................ 547

III. Bestellung durch reine Unterschriftsbeglaubigung . .......... 565

VIII 
Inhaltsübersicht

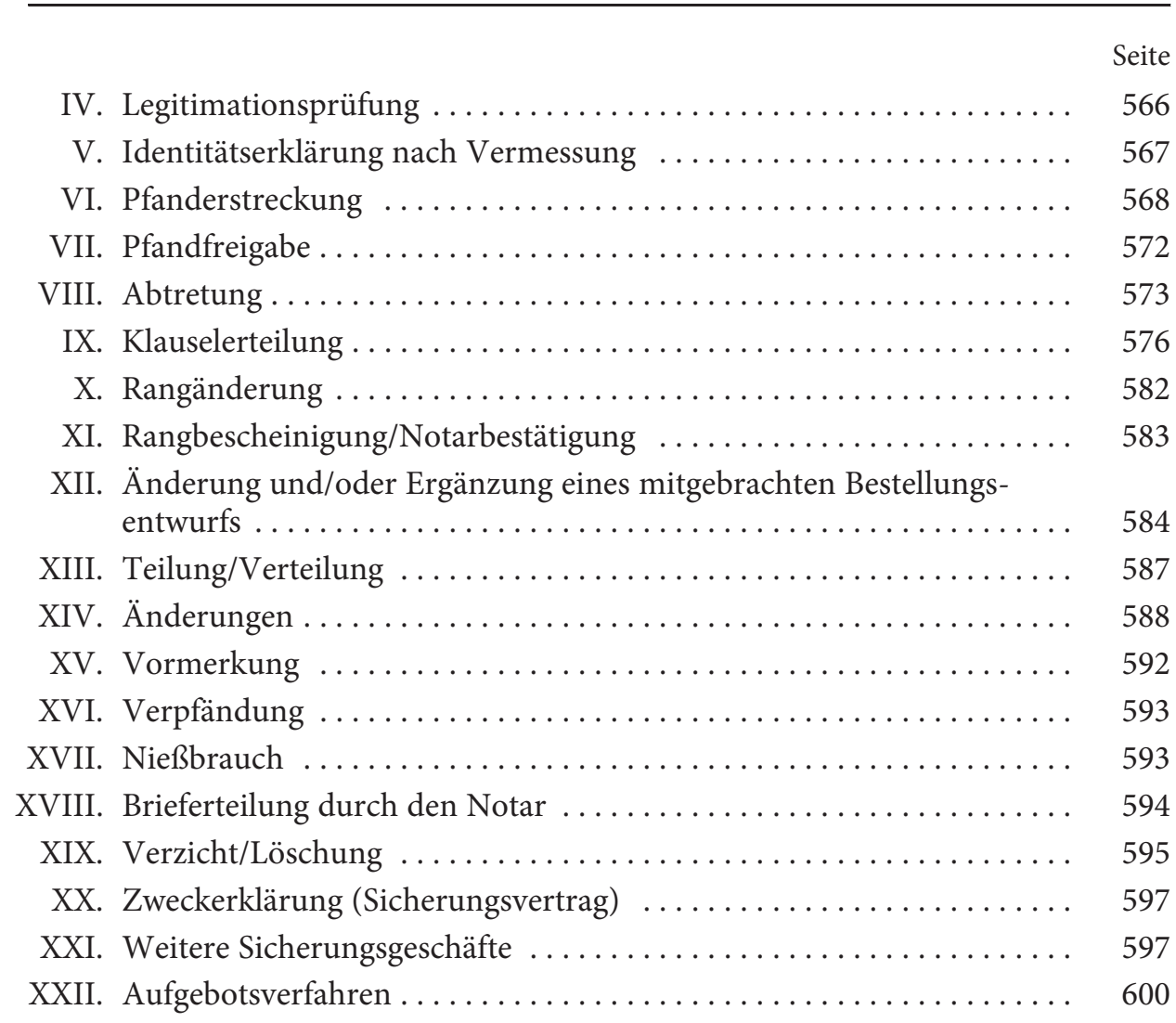

\section{Teil 7 Dienstbarkeiten}

I. Überblick .................................. 606

II. Dienstbarkeiten ................................ 613

\section{Teil 8 Grundbucherklärungen und Grundbuch- einsicht}

I. Überblick ..................................... 653

II. Grundstücksteilung, Grundstücksverbindung . . . . . . . . . . 657

III. Nachbarschaftsrechte .............................. 659

IV. Isolierte Grundbuchanträge zu Miteigentümervereinbarungen . . . . 660

V. Löschungs-, Aufgabe- und Aufhebungserklärungen ............ 663

VI. Grundbuchberichtigungserklärungen .................. 670

VII. Pfanderstreckung, Pfandfreigabe, Rangänderung $\ldots \ldots \ldots \ldots \ldots .678$

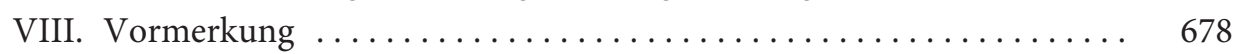


IX. Zeugnisse zum Nachweis der Auseinandersetzung eines Nachlasses

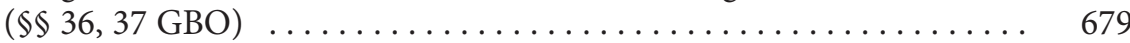

X. Optionen (Ankaufsrechte, Vorkaufsrechte, Wiederkaufsrechte) . . . . 679

XI. Isolierte Grundbucheinsicht, Grundbuchabdruck, Datei . . . . . . . . . 679

\section{Teil 9 Vorzeitige Beendigung des Beurkundungs- verfahrens, Entwurf, Beratung}

I. Überblick ....................................... 686

II. Vorzeitige Beendigung des Beurkundungsverfahrens . . . . . . . . . 701

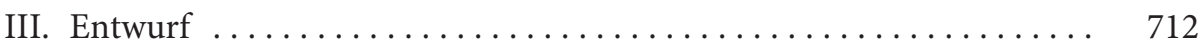

IV. Beratung .............................. 720

\section{Teil 10 Vollmachten und Zustimmungen allgemein}

I. Überblick .................................. 731

II. Vollmacht durch Beurkundung oder Entwurf mit Unterschriftsbeglaubigung für ein bestimmtes Geschäft .............. 736

III. Vollmacht durch Beurkundung oder Entwurf mit Unterschriftsbeglaubigung für Geschäfte allgemeiner Art $\ldots \ldots \ldots \ldots \ldots \ldots .743$

IV. Vollmacht - reine Unterschriftsbeglaubigung ............. 746

V. Vollmachtsbestätigung durch Beurkundung oder Entwurf mit Unterschriftsbeglaubigung . . . . . . . . . . . . . . . . . . 747

VI. Höchstwert einer Vollmacht . . . . . . . . . . . . . . . . . . . . . . 748

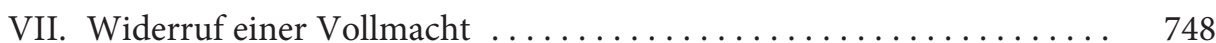

VIII. Mehrheit von Vollmachten . . . . . . . . . . . . . . . . . . . . . . . . $\quad 750$

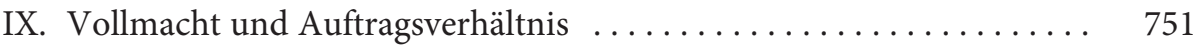

X. Zustimmung durch Beurkundung oder Entwurf mit Unterschrifts-

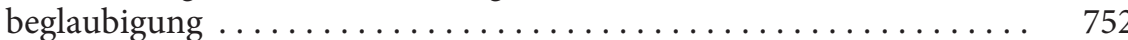

XI. Zustimmung - reine Unterschriftsbeglaubigung . . . . . . . . . . . . . 759

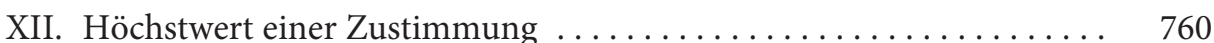

\section{Teil 11 Unterschriftsbeglaubigungen}

I. Überblick .................................. 763

II. Unterschriftsbeglaubigungen unter bestimmte Erklärungen . . . . . . 768

III. Mehrere getrennte Unterschriftsbeglaubigungen auswärts . . . . . . . . 780

IV. Unterschriftsbeglaubigung unter mehrere Erklärungen . . . . . . . 782

V. Nachträgliche Unterschriftsbeglaubigung . . . . . . . . . . . . . 789

$X$ 
Inhaltsübersicht

Seite

\section{Teil 12 Abschriftsbeglaubigungen}

I. Überblick . . . . . . . . . . . . . . . . . . . . . . . . . . . . . . . . . . . 791

II. Ausgewählte Abschriftsbeglaubigungen . . . . . . . . . . . . . 794

\section{Teil 13 Tatsachenbescheinigungen}

I. Überblick . . . . . . . . . . . . . . . . . . . . . . . . . 804

II. Ausgewählte Tatsachenbescheinigungen $\ldots \ldots \ldots \ldots \ldots \ldots \ldots . \ldots 8$

\section{Teil 14 Aufnahme von Wechsel- und Scheckprotesten}

I. Überblick . . . . . . . . . . . . . . . . . . . . . . . . . . . . . . . . 823

II. Aufnahme von Wechsel- und Scheckprotesten .............. 825

\section{Teil 15 Vermögensverzeichnisse}

I. Überblick .................................. 829

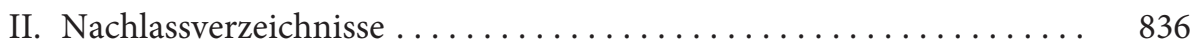

III. Sonstige Vermögensverzeichnisse . . . . . . . . . . . . . . . . . 839

IV. Vorzeitige Beendigung des Verfahrens ................ 841

V. Vermögensverzeichnis als Teil des Vertrages .............. 843

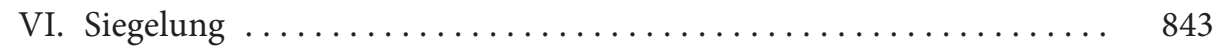

VII. Mitwirkung des Notars als Urkundsperson ................. 845

\section{Teil 16 Verlosungen}

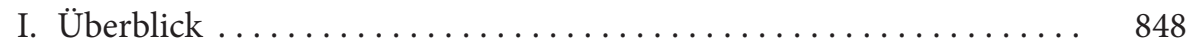

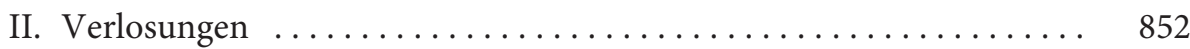

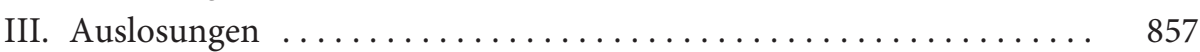

IV. Beurkundung des Verlosungsherganges ................ 859

V. Vorzeitige Beendigung des Verfahrens ................... 859

\section{Teil 17 Eide und eidesstattliche Versicherungen}

I. Überblick ....................................... 862

II. Eidesstattliche Versicherungen gegenüber einer Behörde . . . . . . . . 869

III. Eidesstattliche Versicherung zur Erlangung eines Zeugnisses ..... 870

IV. Eidesstattliche Versicherung zur Erlangung eines Erbscheins oder eines Europäischen Nachlasszeugnisses $\ldots \ldots \ldots \ldots \ldots \ldots \ldots \ldots . \ldots 73$ 
V. Vorzeitige Beendigung des Verfahrens ................. 873

VI. Prioritätsverhandlung . . . . . . . . . . . . . . . . . . . . 873

VII. Eide ................................. 874

VIII. Vernehmung von Zeugen und Sachverständigen $\ldots . \ldots \ldots \ldots \ldots \ldots$. . . 874

\section{Teil 18 Beurkundungen im Kindschaftsrecht}

I. Überblick . . . . . . . . . . . . . . . . . . . . . . . . . . . . . . . 877

II. Beurkundungen von Erklärungen nicht miteinander verheirateter Eltern ............................... 881

III. Annahme als Kind $\ldots \ldots \ldots \ldots \ldots \ldots \ldots \ldots \ldots \ldots \ldots \ldots$

IV. Künstliche Befruchtung und Kinderwunschverträge . . . . . . . . . . 891

V. Einbenennung gem. $₫ 1618$ BGB ... . . . . . . . . . . . . . . 894

\section{Teil 19 Erbrechtliche Vorgänge}

I. Überblick . . . . . . . . . . . . . . . . . . . . . . . . . . 903

II. Beurkundung eines Testaments . . . . . . . . . . . . . . . . . . 917

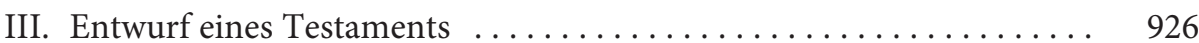

IV. Bestimmte Vollzugstätigkeiten ................... 928

V. Erbverträge und Ehe- und Erbverträge . . . . . . . . . . . . . . . . 929

VI. Rückgabe eines Erbvertrags aus der notariellen Verwahrung . . . . . . . 939

VII. Stornierung von Verfügungen von Todes wegen

(Aufhebung, Rücktritt, Widerruf) . . . . . . . . . . . . . . . . . . . . . . 940

VIII. Erb- und Pflichtteilsverzichte ....................... 948

IX. Erbausschlagung und Anfechtung . . . . . . . . . . . . . . . 952

X. Eidesstattliche Versicherung zur Erlangung eines Erbscheins . . . . . 956

XI. Erbauseinandersetzung . . . . . . . . . . . . . . . . . . . 973

XII. Erbschaftskauf . . . . . . . . . . . . . . . . . . . . . . . . . . 980

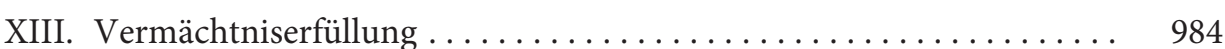

XIV. Erbrechtliche Auslegungsverträge . . . . . . . . . . . . . . 986

XV. Nachlassverzeichnis $\ldots \ldots \ldots \ldots \ldots \ldots \ldots \ldots \ldots \ldots \ldots . \ldots \ldots 6$

\section{Teil 20 Eheverträge und Scheidungsvereinbarungen}

I. Überblick . . . . . . . . . . . . . . . . . . . . . . . . . . . . . . . . . . . . . 993

II. Gütertrennung $\ldots \ldots \ldots \ldots \ldots \ldots \ldots \ldots \ldots \ldots \ldots \ldots \ldots \ldots \ldots$

III. Modifizierung der Zugewinngemeinschaft . . . . . . . . . . . . . . . . 1014 
Inhaltsübersicht

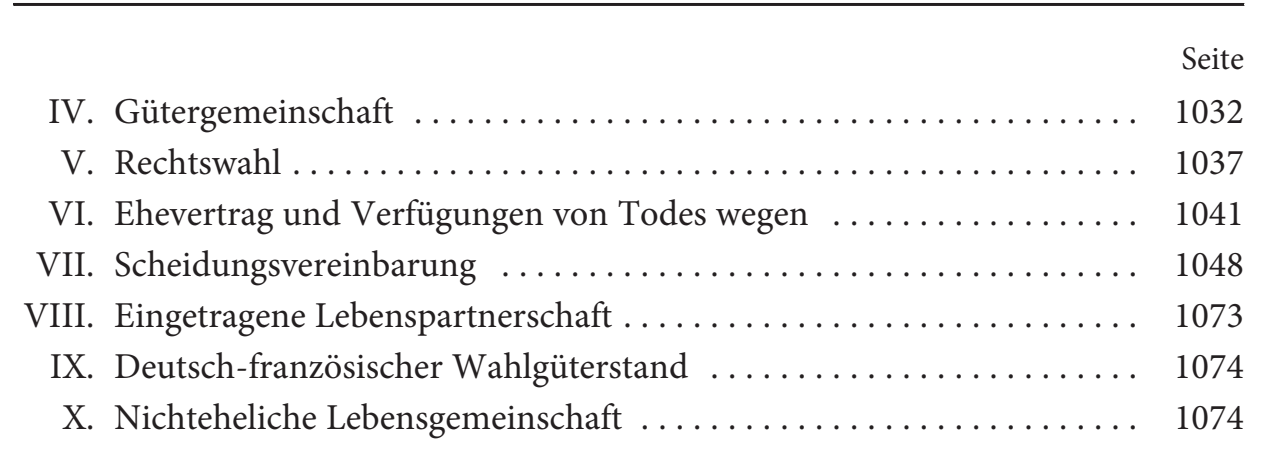

\section{Teil 21 Gesellschaftsrecht}

I. Überblick ..................................... 1097

II. Kostenrechtlicher Wert von Beteiligungen und Anteilen an Gesellschaften anhand der Bilanz .................. 1113

III. Gesellschaft bürgerlichen Rechts $(\mathrm{GbR}) \ldots \ldots \ldots \ldots \ldots \ldots \ldots \ldots \ldots$

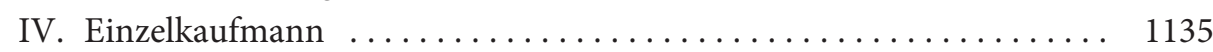

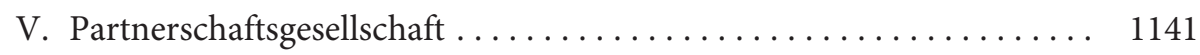

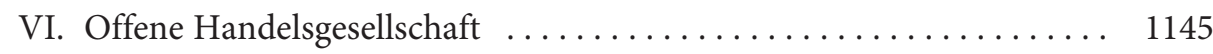

VII. Kommanditgesellschaft ...................... 1158

VIII. Verein ................................. 1174

IX. Anmeldung zum Genossenschaftsregister $\ldots \ldots \ldots \ldots \ldots \ldots \ldots \ldots . \ldots 1181$

X. Gesellschaft mit beschränkter Haftung . . . . . . . . . . . . . . . . . . 1184

XI. Aktiengesellschaft .............................. 1273

XII. Zweigniederlassungen $\ldots \ldots \ldots \ldots \ldots \ldots \ldots \ldots \ldots \ldots \ldots \ldots \ldots \ldots . \ldots \ldots 29 . \ldots \ldots$

XIII. Stiftungen $\ldots \ldots \ldots \ldots \ldots \ldots \ldots \ldots \ldots \ldots \ldots \ldots \ldots \ldots \ldots \ldots \ldots \ldots$

XIV. Anmeldungen ohne wirtschaftliche Bedeutung . . . . . . . . . . . 1297

XV. Beherrschungs- und Gewinnabführungsverträge . . . . . . . . . . . 1298

XVI. Beteiligungs- und Gesellschaftervereinbarungen . . . . . . . . . . . . 1307

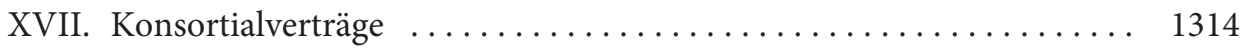

XVIII. Poolvereinbarungen $\ldots \ldots \ldots \ldots \ldots \ldots \ldots \ldots \ldots \ldots \ldots \ldots \ldots \ldots$

\section{Teil 22 Umwandlungsrecht mit Anmeldungen}

I. Überblick ... . . . . . . . . . . . . . . . . . . . . . . . . . . . . . . . . . 1324

II. Aktivwert von Gesellschaften nach kostenrechtlichen Gesichts-
punkten $\ldots \ldots \ldots \ldots \ldots \ldots \ldots \ldots \ldots \ldots \ldots \ldots \ldots \ldots \ldots \ldots \ldots \ldots \ldots \ldots \ldots \ldots \ldots \ldots$

III. Wert bestimmter Gesellschaftsanteile $(\$ 54$ GNotKG $) \ldots \ldots \ldots \ldots . . \ldots 335$

IV. Umwandlungsvorgänge $\ldots \ldots \ldots \ldots \ldots \ldots \ldots \ldots \ldots \ldots \ldots \ldots \ldots \ldots$ 
Seite

\section{Teil 23 Vorsorgeverfügungen}

I. Überblick . . . . . . . . . . . . . . . . . . . . . . . . . . . . . . . . . . . . . . . 1404

II. Vorsorgevollmacht durch Beurkundung oder Entwurf mit Unterschriftsbeglaubigung ...................... 1410

III. Vorsorgevollmacht - reine Unterschriftsbeglaubigung . . . . . . . 1417

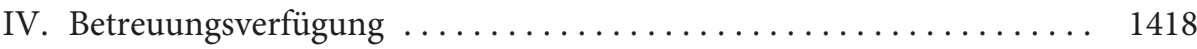

V. Patientenverfügung ............................. 1420

VI. Betreuungs- und Patientenverfügung ................... 1422

VII. Höchstwert einer Vorsorgevollmacht . . . . . . . . . . . . . . . . . . . 1423

VIII. Widerruf einer Vorsorgevollmacht ..................... 1424

IX. Mehrheit von Vorsorgevollmachten/Gegenseitige Vorsorgevollmachten ................................ 1427

X. Vorsorgevollmacht mit Betreuungs- und Patientenverfügung . . .... 1428

XI. Vorsorgeurkunde für elektronische Zugangsberechtigung ........ 1431

\section{Teil 24 Vollstreckbarerklärungen}

I. Überblick ..................................... 1434

II. Vollstreckbarerklärungen ......................... 1436

\section{Teil 25 Freiwillige Versteigerung von Grund- stücken}

I. Überblick ..................................... 1446

II. Verfahrensgebühr ................................... 1449

III. Aufnahme einer Schätzung . . . . . . . . . . . . . . . . . . . . . . 1449

IV. Abhaltung des Versteigerungstermins . .................. 1450

V. Freiwillige Versteigerung durch einen privaten Auktionator ....... 1452

VI. Ausbietungsgarantie ............................. 1456

\section{Teil 26 Tätigkeiten im Zusammenhang mit Schiffen}

I. Überblick .................................... 1459

II. Anmeldungen zum Schiffs-/Schiffsbauwerkregister . . . . . . . . . . . 1465

III. Schiffshypothek ................................... 1467

IV. Veräußerung eines Schiffs $\ldots \ldots \ldots \ldots \ldots \ldots \ldots \ldots \ldots \ldots \ldots . \ldots \ldots$ 
Inhaltsübersicht

Seite

Teil 27 Auslagen und elektronischer Vollzug

I. Überblick ... . . . . . . . . . . . . . . . . . . . . . . . . . . . . . . . . . . 1474

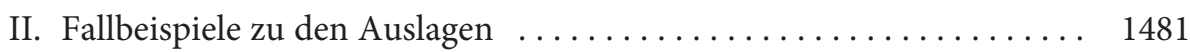

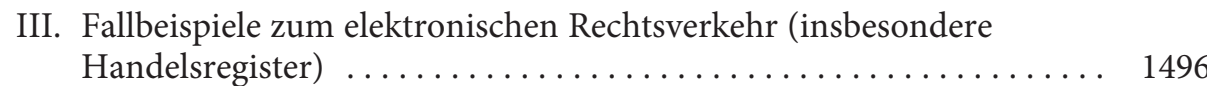

IV. Fallbeispiele zum elektronischen Rechtsverkehr (Grundbuchamt) . . 1502

\section{Teil 28 Zusatzgebühren}

I. Überblick ... . . . . . . . . . . . . . . . . . . . . . . . . . . . . . . . . . 1513

II. Beurkundungen zur Unzeit und/oder außerhalb der

Geschäftsstelle .................................... 1517

III. Tätigkeiten in fremder Sprache $\ldots \ldots \ldots \ldots \ldots \ldots \ldots \ldots \ldots \ldots . \ldots \ldots 23$

Teil 29 Freiwilliges Umlegungsverfahren

I. Überblick . . . . . . . . . . . . . . . . . . . . . . . . . . 1527

II. Freiwilliges Umlegungsverfahren: Fälle $\ldots \ldots \ldots \ldots \ldots \ldots \ldots \ldots \ldots$

\section{Teil 30 Verwahrung}

I. Verwahrung von Geld, Wertpapieren und Kostbarkeiten ........ 1534

II. Verwahrung von anderen Gegenständen ................ 1535

Teil 31 Vereinbarung über die Kosten - Öffentlichrechtlicher Vertrag nach § 126 GNotKG

I. Allgemeines .................................. 1538

II. Fallbeispiele .................................. 1542

\section{Teil 32 Erschließungsverträge}

I. Überblick .................................. 1545

II. Fallbeispiele .................................. 1548

\section{Teil 33 Miet- und Pachtverträge}

I. Überblick ....................................... 1552

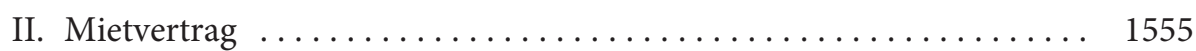

III. Pachtvertrag . . . . . . . . . . . . . . . . . . . . . . . 1560 


\section{Teil 34 Vergleiche}

I. Überblick . . . . . . . . . . . . . . . . . . . . . . . . . . . . . . . . . 1561

II. Beurkundungen . . . . . . . . . . . . . . . . . . . . . 1564

Stichwortverzeichnis $\ldots \ldots \ldots \ldots \ldots \ldots \ldots \ldots \ldots \ldots \ldots \ldots \ldots \ldots \ldots$ 